\title{
Recovery of Background Structures in Nanoscale Helium Ion Microscope Imaging
}

\author{
Alfred S. Carasso and András E. Vladár \\ National Institute of Standards and Technology, \\ Gaithersburg, MD 20899 \\ alfred.carasso@nist.gov \\ andras.vladar@nist.gov
}

This paper discusses a two step enhancement technique applicable to noisy Helium Ion Microscope images in which background structures are not easily discernible due to a weak signal. The method is based on a preliminary adaptive histogram equalization, followed by ‘slow motion' low-exponent Lévy fractional diffusion smoothing. This combined approach is unexpectedly effective, resulting in a companion enhanced image in which background structures are rendered much more visible, and noise is significantly reduced, all with minimal loss of image sharpness. The method also provides useful enhancements of scanning charged-particle microscopy images obtained by composing multiple drift-corrected 'fast scan' frames. The paper includes software routines, written in Interactive Data Language (IDL), ${ }^{1}$ that can perform the above image processing tasks.

Key words: adaptive histogram equalization; background recovery; composed fast-scan frames; HIM; nanoscale helium ion microscopy; noise reduction; progressive fractional diffusion smoothing; SEM.

Accepted: December 15, 2014

Published: December 31, 2014

http://dx.doi.org/10.6028/jres.119.030

\section{Introduction}

The advantages of Helium ion microscopy (HIM) over conventional scanning electron microscopy (SEM) are explored in [9] and [10]. HIM imaging achieves higher resolution with higher contrast and greater depth of field than is possible with SEM. In many cases, HIM images reveal greater surface detail. The secondary electrons that carry the sample's surface detail information are generated in higher quantities by He irradiation than is the case with electron beams. As a result, low probe currents, on the order of one picoampere, can be used effectively to acquire HIM images. The use of small He ion beam currents is also necessary to minimize damage to the sample. However, such low current HIM images are often noisy, and important background structures may not be easily discernible due to the weak signal.

This paper discusses a two-step enhancement technique that can significantly improve this situation. As shown in Fig. 1, this technique results in a companion enhanced image in which deep background structural details are recovered, while noise is significantly reduced with minimal loss of image sharpness. Previous work on denoising HIM imagery [1], did not address the background recovery problem. While limited success was achieved in more recent work on forensic latent fingerprint enhancement [2], the present results, involving an entirely different class of images, could not be anticipated and had to be discovered independently.

The two stage approach used here involves a preliminary image-specific adaptive histogram equalization of the given HIM image [11]. Such equalization enhances background information while significantly magnifying noise, and is not generally advisable with noisy data. However, at the second

\footnotetext{
${ }^{1}$ Mention of commmercial products or services in this report does not imply NIST approval or endorsement of these products or services, nor does it imply that such products or services are necessarily the best available for the intended purpose.
} 


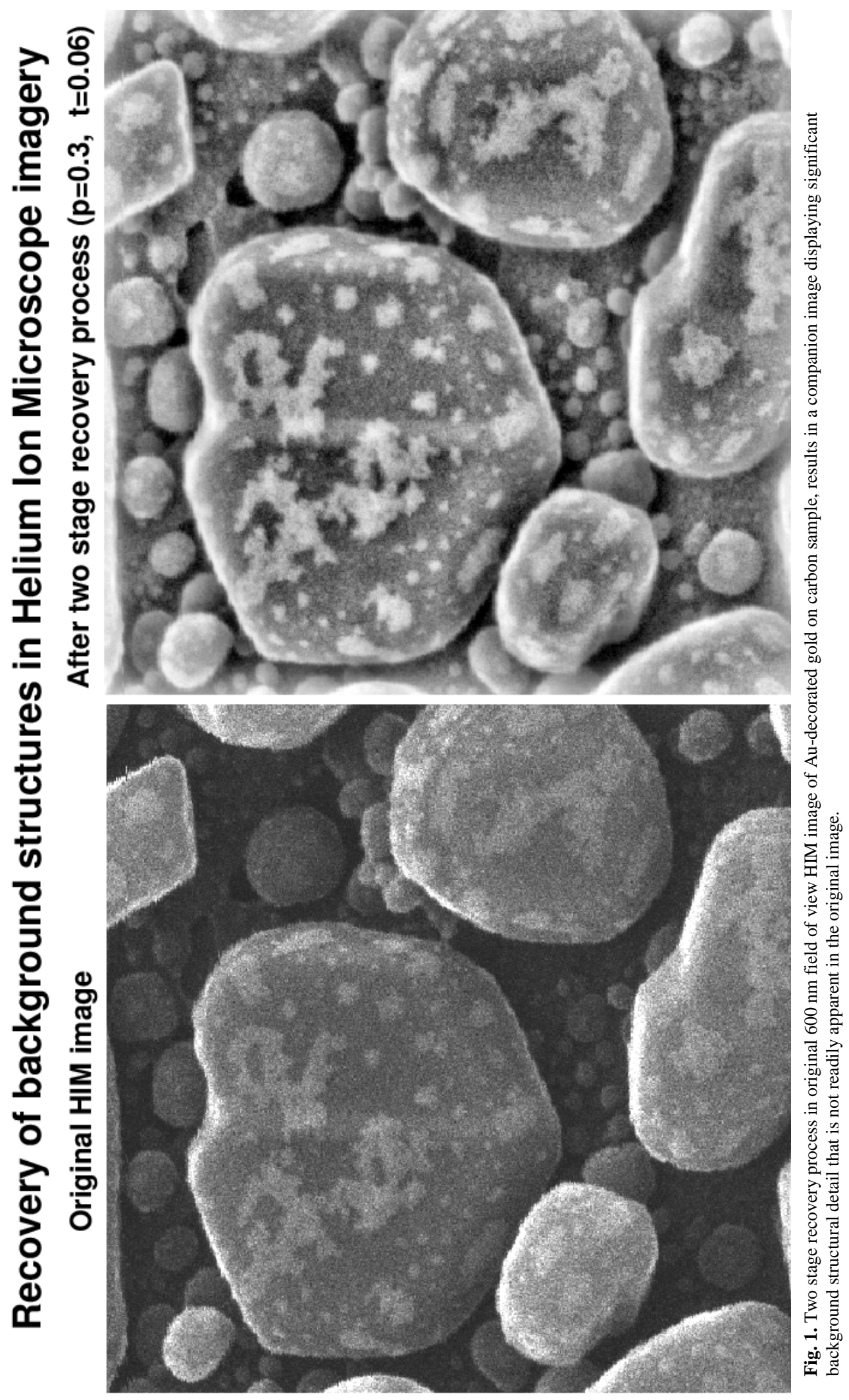


stage, an effective and easy to use progressive denoising technique, based on low exponent Lévy fractional diffusion smoothing, can be successfully applied to this histogram equalized image with magnified noise. As may be inferred from Figs. 2 and 3, such Lévy smoothing can be fine-tuned interactively so as to preserve the detailed surface morphology of the sample. Despite many parameter trials, comparable fidelity to surface detail was not found feasible with some other better-known denoising techniques, such as methods based on minimizing the image 'total variation' norm [6], [8], or methods based on thresholding curvelet transforms [13], [14]. This is illustrated in Figure 4.

The ability to explore efficiently in parameter space and visually select the best result, is the key to successful digital image enhancement. There is no single set of parameters that will be useful in all cases, nor is there an automatic way of selecting the best set of parameters. Only a trained and experienced human analyst can locate useful parameter values, and determine the proper amount of fine tuning that best displays the information sought. The paper includes software routines, written in Interactive Data Language (IDL) [7], that can be used to perform the above two separate image processing tasks.

\section{Preliminary Enhancement of HIM Image Using 'IDLAdapt.pro'}

Histogram equalization is a useful enhancement technique for images where significant information is suspected of being hidden in dark regions. A complete discussion of this technique is given in [11], together with examples illustrating the advantages of the more sophisticated adaptive formulation. However, in practice, usefully recovered background information is often obscured by the amplification of the accompanying noise, and the resulting improvements may not be particularly helpful without additional intervention.

The routine IDLAdapt.pro listed in Sec. 7, is to be applied to an 8 bit greyscale TIFF image, which may be rectangular. The routine identifies and prints the dimensions of that image, performs adaptive histogram equalization, and displays the equalized image. Next, the equalized image is embedded in a zero padded larger square array of even dimension specified by the user. That dimension should typically be between fifteen and twenty percent greater than the larger side of the input image. The zero padded equalized 8 bit greyscale TIFF image is returned in the file 'idlsharp.tiff'. This process is illustrated in Fig. 5.

\section{Progressive Low Exponent Lévy Fractional Diffusion Smoothing}

Given a noisy image $f(x, y)$, the smoothing procedure results from solving an initial value problem for a special type of diffusion equation, with the image $f(x, y)$ as initial data. Such smoothing is applied to the whole image, and not just to a selected portion of the image. With fixed $p$ with $0<p \leq 1$, consider the linear fractional diffusion initial value problem in $L^{2}\left(R^{2}\right)$,

$$
w_{t}=-(-\Delta)^{p} w, t>0, \quad w(x, y, 0)=f(x, y),
$$

where $\Delta$ denotes the 2D Laplacian. This reduces to the classical heat conduction equation when $p=1$. However, our smoothing procedure uses values of $p \ll 1$, such as $p=0.2$, for example. Define the $2 \mathrm{D}$ Fourier transform of the image $f(x, y)$ by

$$
\mathcal{F}\{f\}=\hat{f}(\xi, \eta) \equiv \int_{R^{2}} f(x, y) \exp \{-2 \pi i(\xi x+\eta y)\} d x d y .
$$

Eq. (1) has the unique Fourier domain solution

$$
\hat{w}(\xi, \eta, t)=\exp \left\{-t\left[(2 \pi \xi)^{2}+(2 \pi \eta)^{2}\right]^{p}\right\} \hat{f}(\xi, \eta), t>0,
$$

from which $w(x, y, t)$ can be found by inverse Fourier transformation 


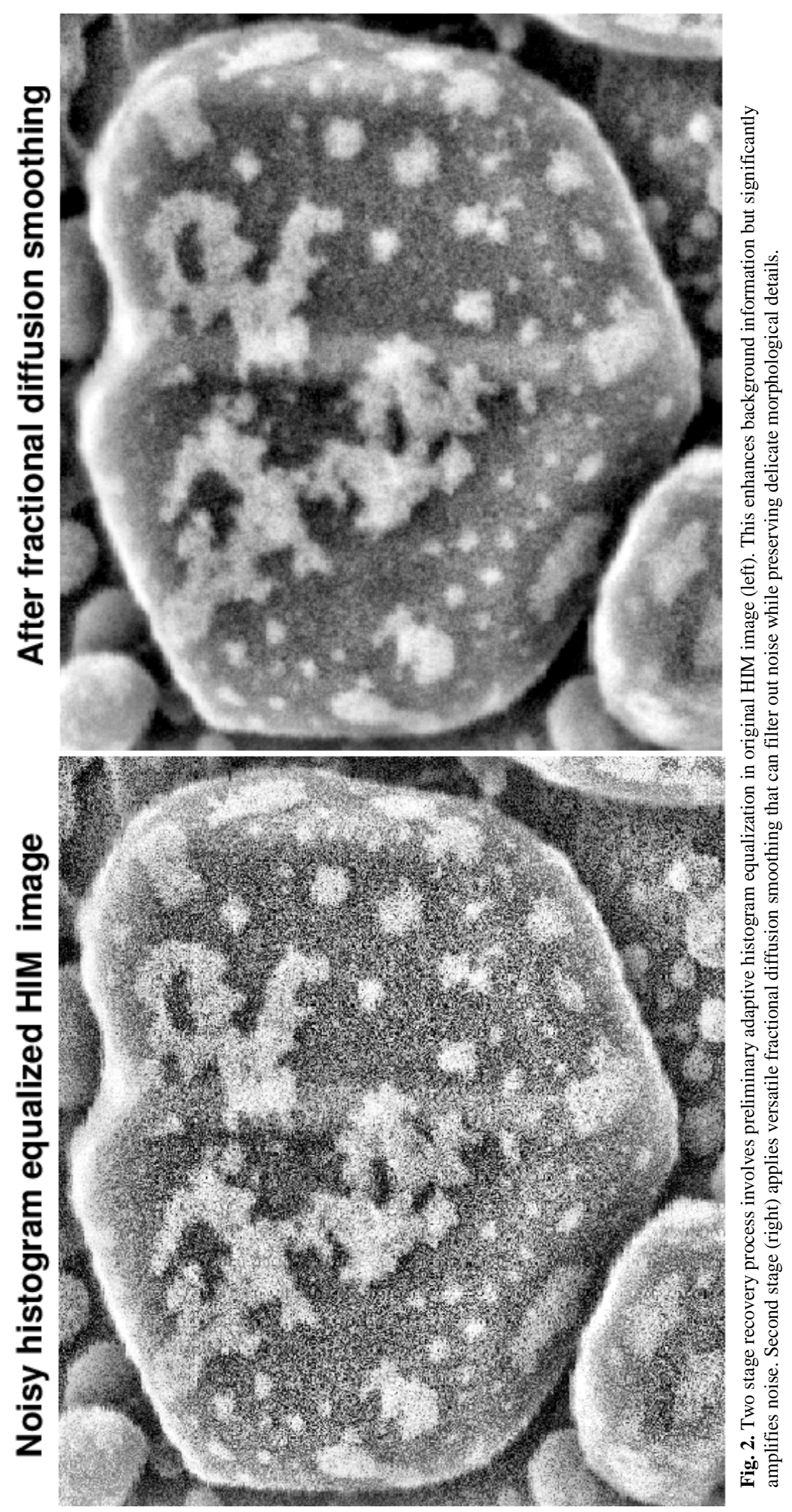




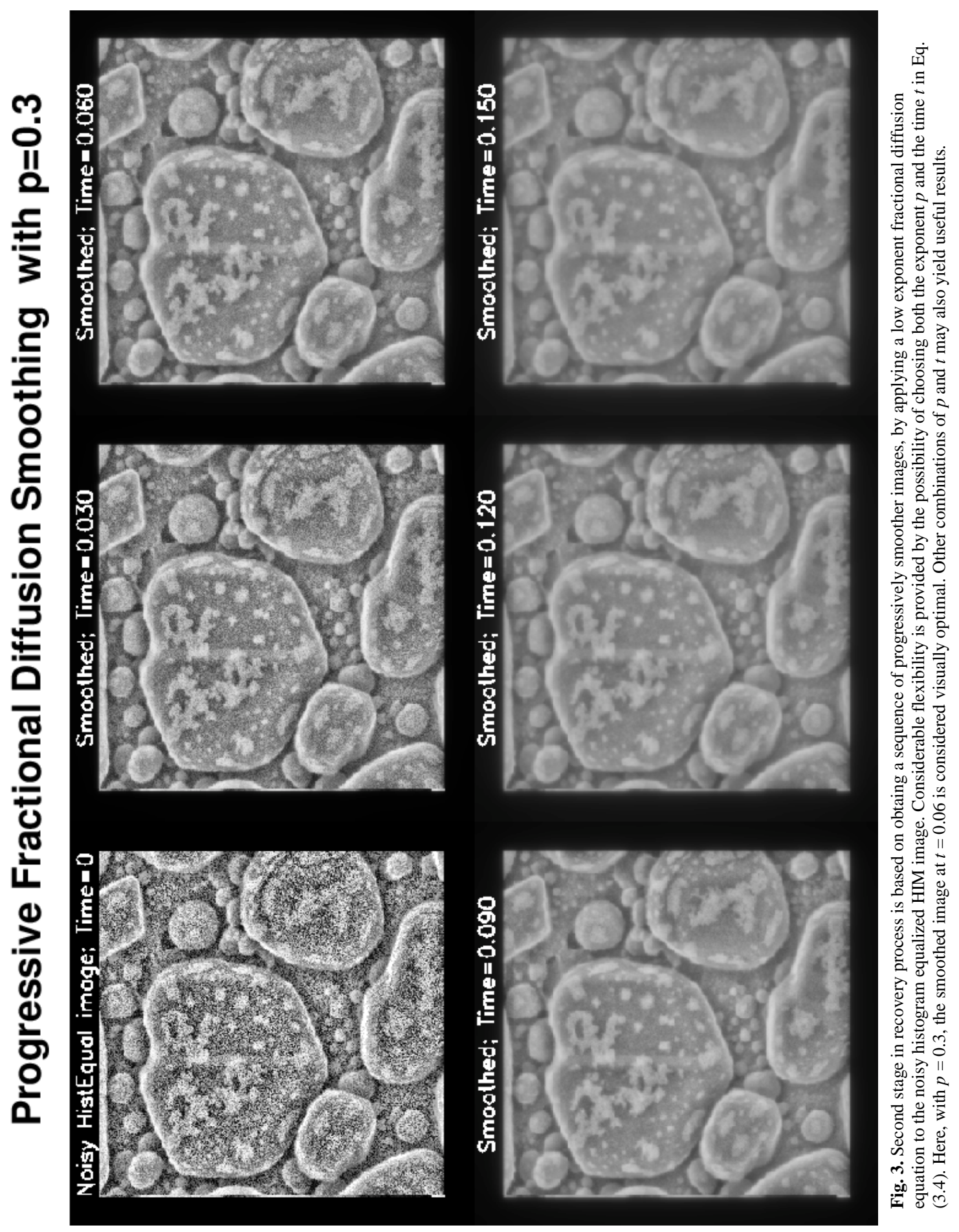



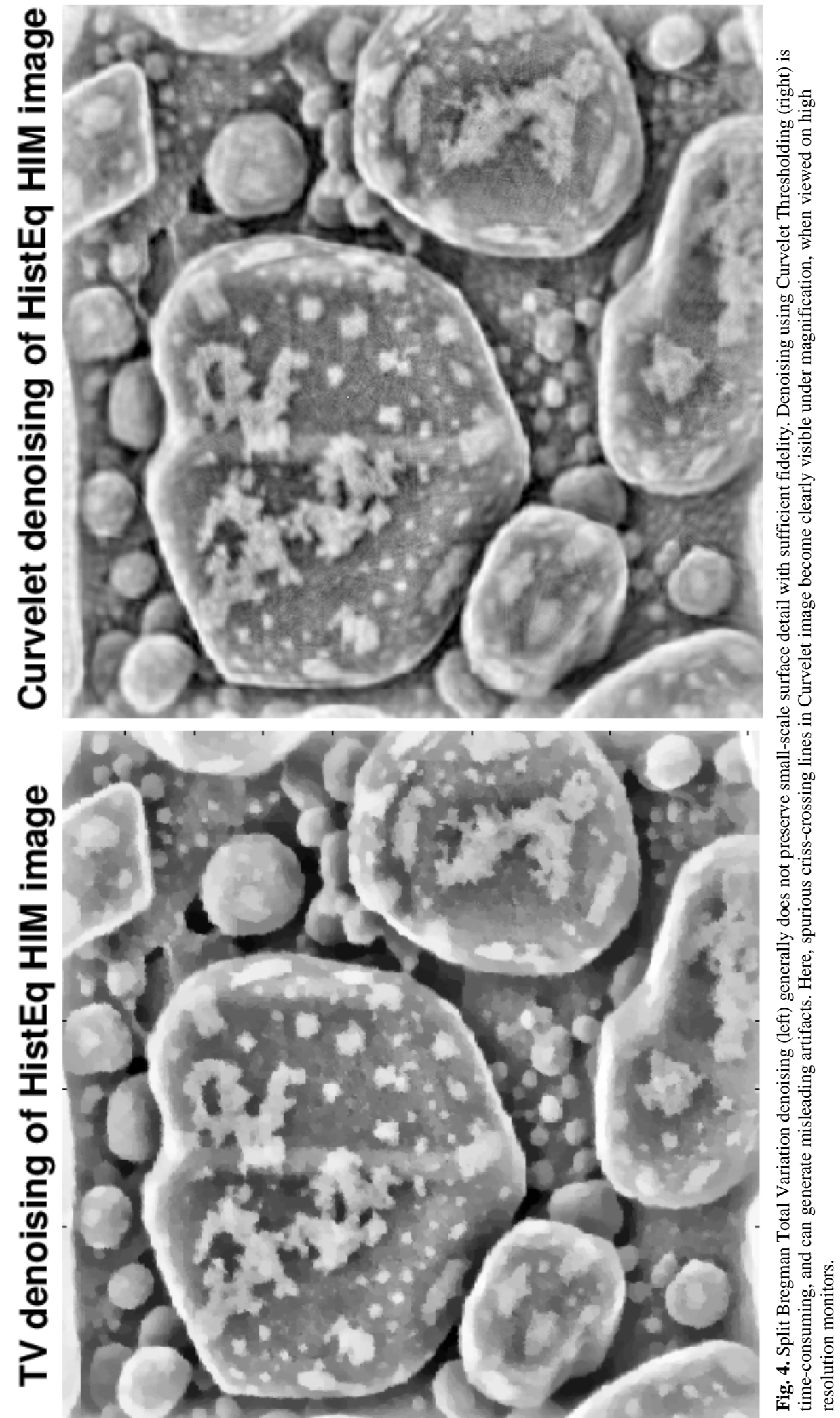


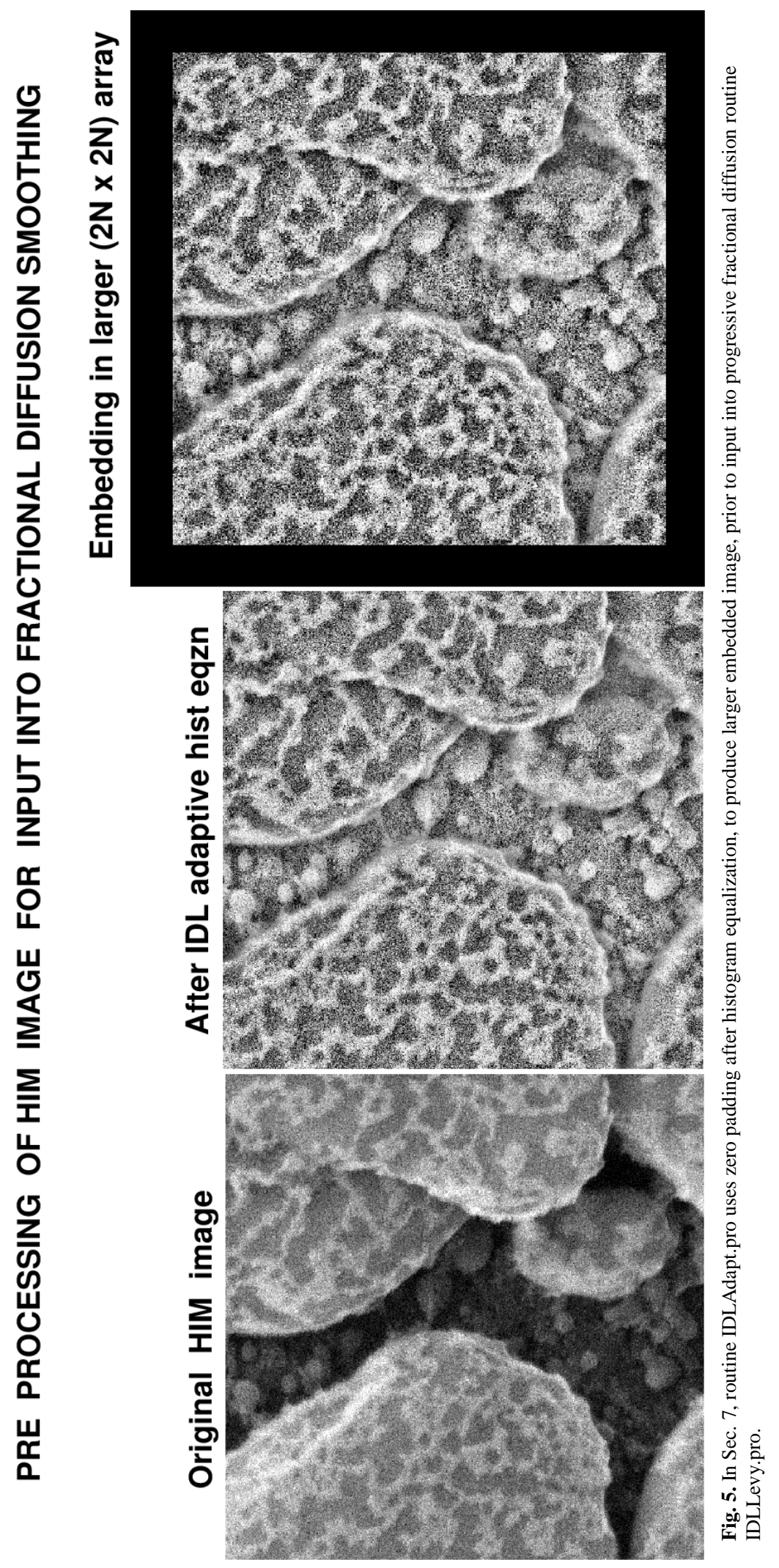




$$
w(x, y, t)=\int_{R^{2}} \exp \{2 \pi i(\xi x+\eta y)\} \exp \left\{-t\left[(2 \pi \xi)^{2}+(2 \pi \eta)^{2}\right]^{p}\right\} \hat{f}(\xi, \eta) d \xi d \eta .
$$

As is evident from Eq. (4), $w(x, y, t)$ becomes increasingly smoother as $t$ increases. However, for small $p$, and over a short time interval, the smoothed image may be expected to retain many of the essential features present in the initial data $f(x, y)$.

In Eq. (3), the function

$$
\hat{h}(\xi, \eta, t)=\exp \left\{-t\left[(2 \pi \xi)^{2}+(2 \pi \eta)^{2}\right]^{p}\right\}, t>0,
$$

is the Fourier transform of the Green's function for the linear fractional diffusion equation in Eq. (1). For each fixed $t>0$, the function in Eq. (5) is also the Fourier transform of an isotropic Lévy stable probability density function with exponent $2 p$, [12]. When $p=1$, Eq. (5) corresponds to a Gaussian distribution. For $p \ll 1$, Eq. (5) corresponds to a heavy-tailed density in physical $(x, y)$ space. Unlike a Gaussian, that Lévy density is not known in closed form in the physical variables $(x, y)$, and it has infinite mean and infinite variance.

Smoothing an image by convolution with a Gaussian is equivalent to using $p=1$, and solving the heat conduction equation in Eq. (1). The significance of Lévy stable fractional diffusion smoothing with $p \ll 1$ can be inferred from Eq. (3). Clearly, attenuation of high frequency information, corresponding to large $(|\xi|+|\eta|)$, is dramatically more severe when $p=1$, than it is when $p=0.1$ for example.

\section{FFT Lévy Fractional Diffusion Smoothing Using 'IDLLevy.pro’}

As illustrated in Fig. 5, the smoothing software routine IDLLevy.pro, listed in Sec. 7, assumes a zero padded square input image of even dimension, and returns a square smoothed image of the same size. The smoothed image can subsequently be cropped to the original size.

Given the $2 N \times 2 N$ pixel image $f(x, y)$ as initial data, 'IDLLevy.pro' computes the solution $w(x, y, t)$ in Eq. (1) at any given $t>0$, by using the forward and inverse FFT to implement the operations in Eq. (3) and Eq. (4) respectively. In order to render mathematical formulae more transparent, we use the same notation, $\hat{f}(\xi, \eta)$, for both discrete and continuous Fourier transforms. In the discrete FFT case, the frequencies $2 \pi \xi$ and $2 \pi \eta$ are understood to be integer-valued and to range from $-N$ to $N$. After selecting a tentative maximum smoothing time $T_{\max }$ at which to terminate the smoothing process, Eq. (4) can be evaluated at finitely many intermediate times $0=t_{0}<t_{1}<t_{2}<t_{3}<\cdots=T_{\text {max }}$, to create a suite of progressively smoother images. In 'IDLLevy.pro', a total of six images are displayed at times $t_{m}=\left\{(m-1) * T_{\max }\right\} / 5, m=1, \ldots, 6$. The first image is the original unsmoothed IDL Histogram image, while the sixth image is the smoothest image at the final time $T_{\max }$. A user may select an image at some $t_{m} \leq T_{\max }$ as the optimal image, or may elect to try a different value of $T_{\max }$. The routine 'IDLLevy.pro' is applied as follows:

- At the prompt, for the Lévy exponent $p$, a value between 0.1 and 0.4 is a good first choice, with the larger value reserved for very noisy images. As a rule, values $\leq 0.2$ should be explored prior to choosing a larger $p$. For final time of smoothing $T_{\max }$, a number between 0.1 and 0.3 should be entered as a good first choice. Exploring several values of $T_{\max }$ is likewise quite useful.

- For each choice of $T_{\max }$, the associated suite of six progressively smoother images is computed and displayed in a matter of a few seconds. The user is then prompted to select the optimal smoothed image by entering a picture number between 2 and 6 . Several trial choices can be explored. For each trial selection of optimal image, the original unsmoothed image and the selected optimal image are displayed side by side.

- When a final selection is made, one exits 'IDLLevy.pro' by entering the number -1 . The suite of six progressively smoother images corresponding to the last choice of $T_{\max }$, is in the file 'LevyEvol.tiff'. The final selection of optimal image in that suite is now in the file 'Levysmooth.tiff'. 


\section{Some Examples of HIM Image Enhancement}

Figures 6 through 9 illustrate the kind of improvements that can be obtained using the above IDL software routines. Note the small values of $t$ in these figures.

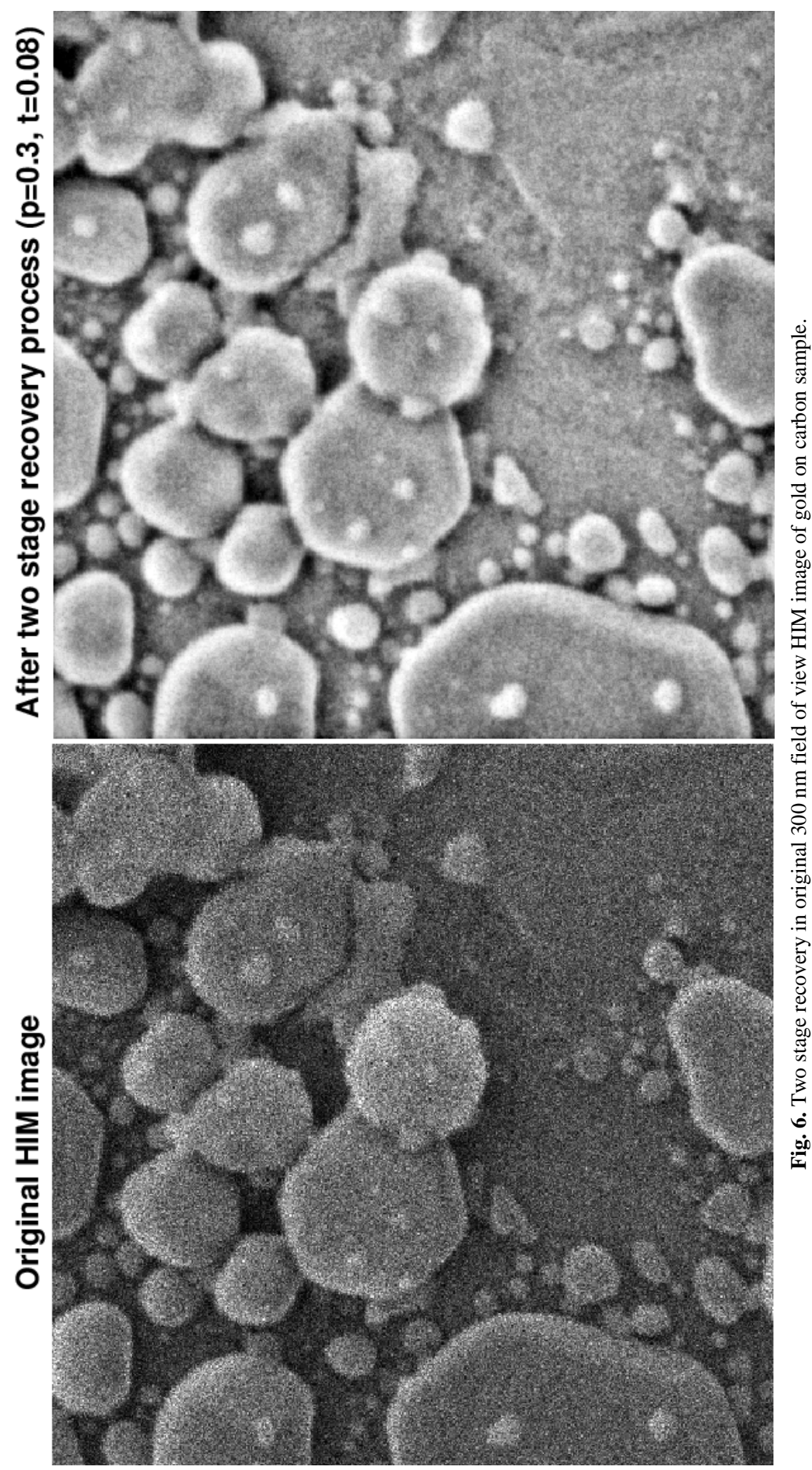




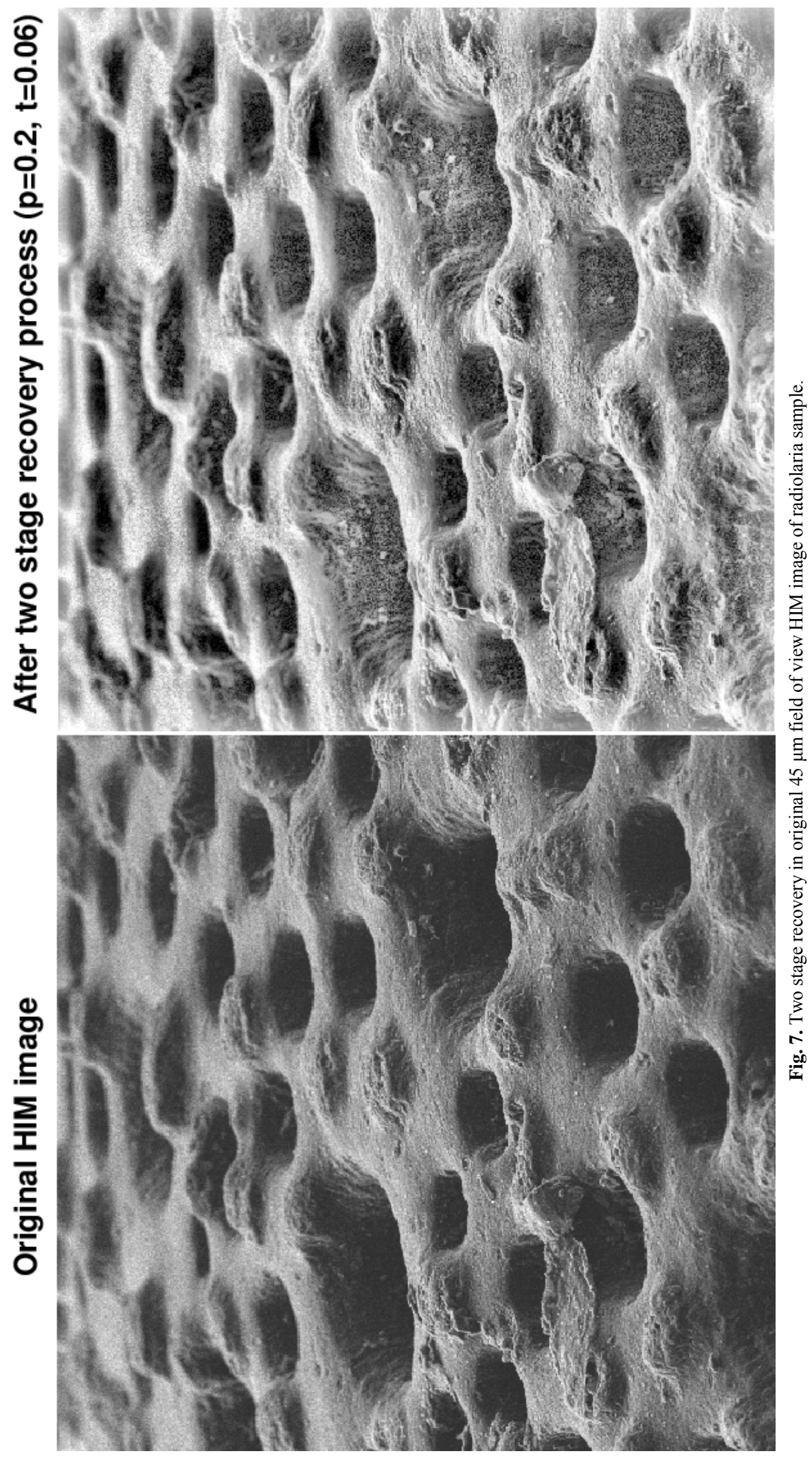




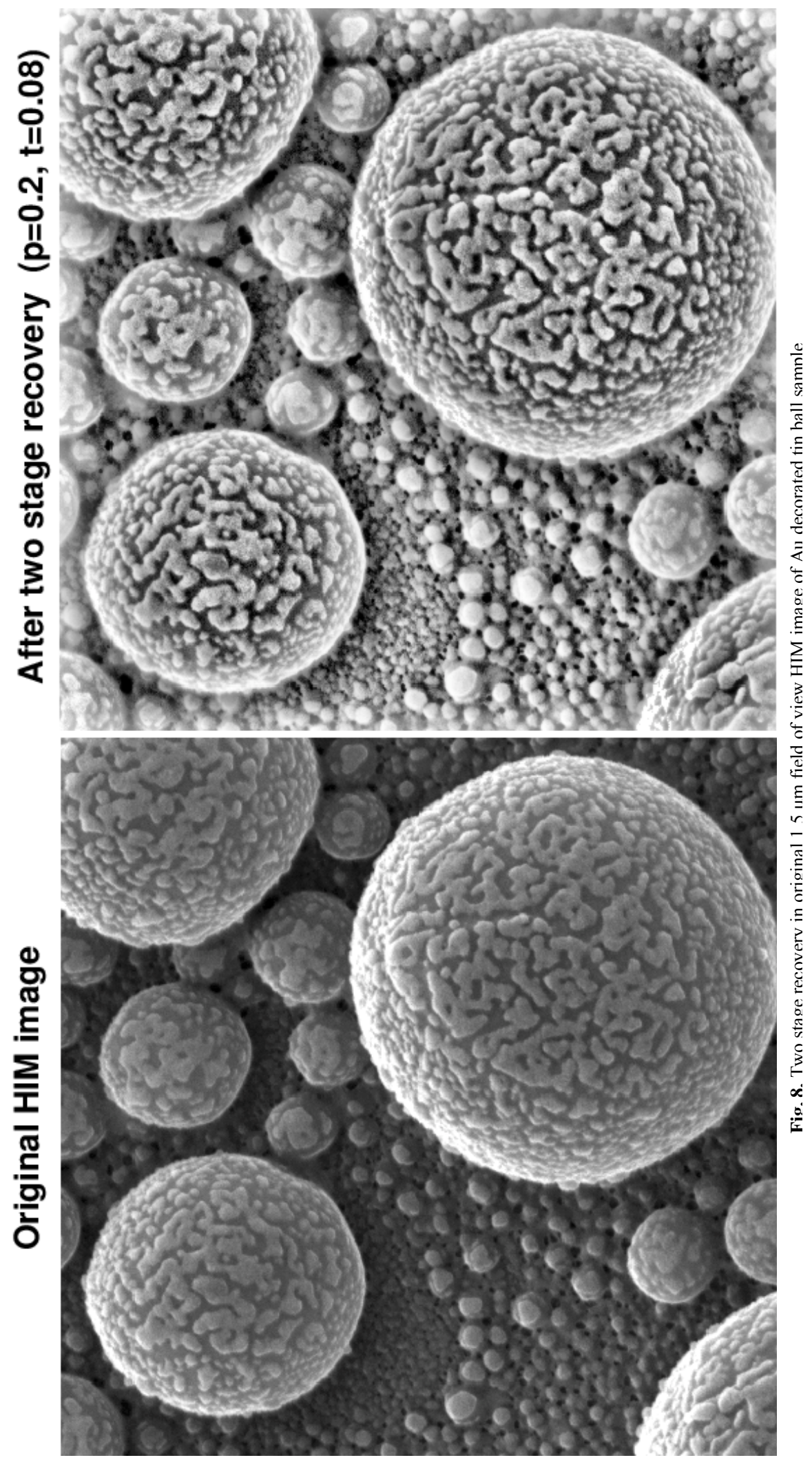




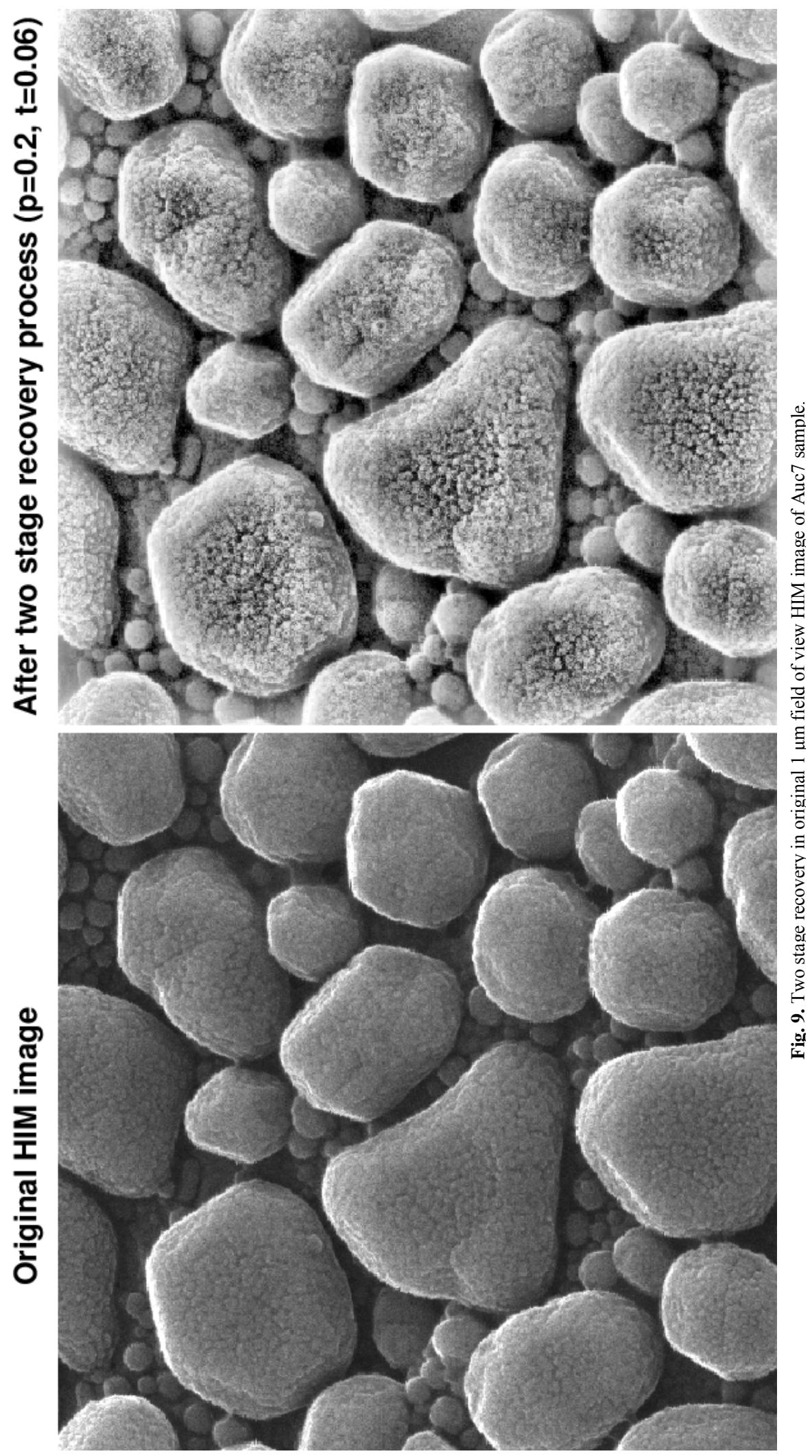




\section{Drift-Corrected Composition of Multiple Fast Scan Frames}

A NIST-developed image composition technique for scanning charged-particle microscopy is discussed in [3-5]. This methodology is based on super-fast acquisition of a large number of image frames. Due to the small beam currents generally used in scanning particle beam microscopy, these individual frames are inherently very noisy, but exhibit significantly less drift-related distortions. The drift correction takes place after finding the center of each frame, properly aligning these frames, and adding them together into a single image. Such composed images contain much less noise and exhibit significantly less blur and deformation than do images obtained by traditional slow scan methods, or images obtained by simply adding together fast image frames without compensating for drift. To improve repeatability of HIM and SEM images, this technique must be used with the minimum number of fast images, which limits the achievable SNR. In many cases, the resulting composed image is still somewhat noisy and some type of noise processing may be beneficial.

As shown in Fig. 10, useful improvements of such composed SEM imagery are possible using the techniques in the present paper. Additionally, improvements to composed HIM imagery are also possible as shown in Fig. 11. The left image in Fig. 11 is a composition of 10 fast image frames. The NIST processed right image in Fig. 11 shows new details, such as augmented faint greyscale transitions at the tops of the gold grains, as well as very fine structures near these tops. Such details can hardly be seen initially in the original left image; however, they become discernible in the original image after studying the companion NIST-enhanced image on the right.

Improvements to single raw HIM image frames are also possible as shown in Fig. 12. One can recognize features in the enhanced right image in Fig. 12 that might easily have been dismissed as noise in the raw image, rather than actual information already present in that raw image.

Because the very finely focused helium ion beam in HIM imaging readily mills the gold particles in Fig. 12, long image acquisition times are not possible without substantially altering the sample. The best solution is the acquisition of a set of fast images that can be composed into a single image, yet one that does not show significant sample modification. This often leads to inherently noisy images. However, this can be remedied using the two-step process discussed in this paper, which results in less noisy images with more perceptible fine details.

\section{Two IDL Routines for HIM Image Enhancement}

The two software routines listed below were used to produce the enhanced images shown in the figures.

\section{IDL ROUTINE FOR ADAPTIVE HISTOGRAM EQUALIZATION}

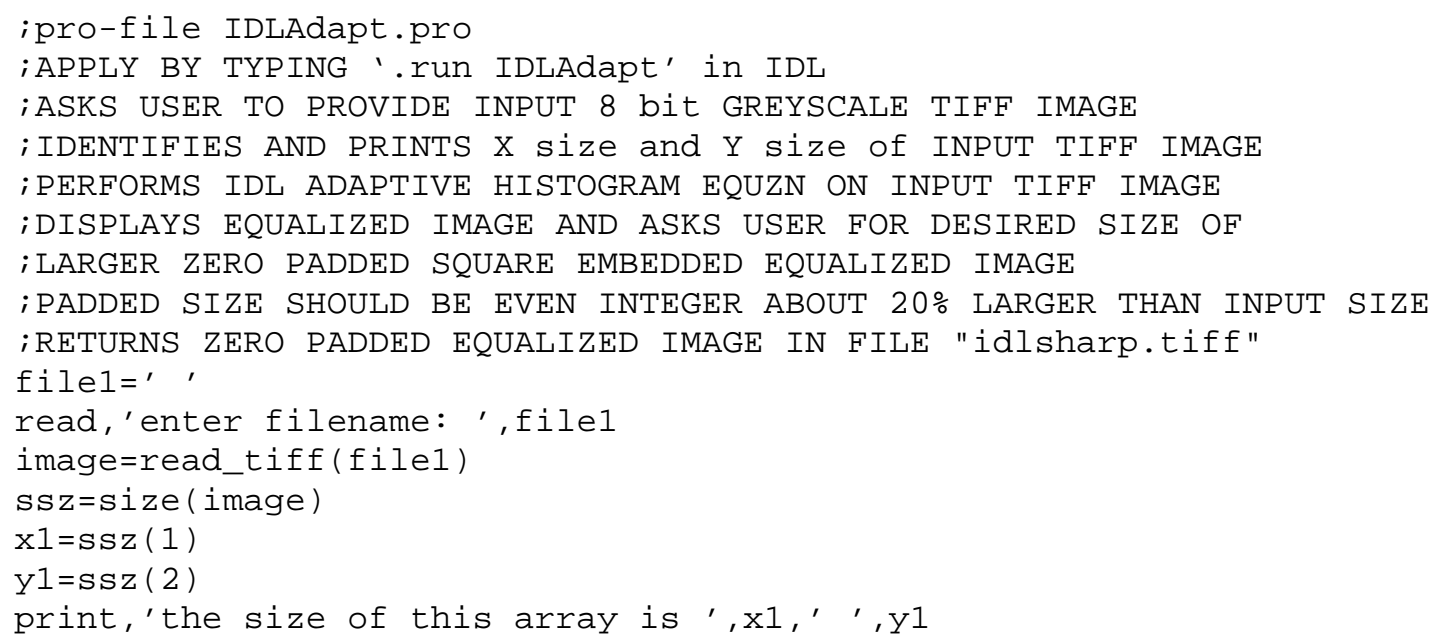


window, $\odot, x$ size $=x 1$, ysize $=y 1$

image=adapt_hist_equal(image)

tvscl, image

$\times 2=0$

$\mathrm{y} 2=0$

read,"enter size of DESIRED ZERO PADDED SQUARE image: ",x2 if $(x 2$ le $\max ([x 1, y 1]))$ then begin print, 'DESIRED IMAGE TOO SMALL' goto, finishup endif

$\mathrm{y} 2=\mathrm{x} 2$

$\mathrm{hn} \times 2=(\mathrm{x} 2-\mathrm{x} 1) / 2$

hny $2=(y 2-y 1) / 2$

image $2=$ bytarr $(x 2, y 2)$

image 2(hnx2: hnx2+x1-1, hny2: hny2+y1-1)=image

window, 1, xsize $=x 2$, ysize $=y 2$

tvscl, image2

write_tiff, 'idlsharp.tiff', image2

finishup:

end

\section{IDL ROUTINE FOR LÉVY FRACTIONAL DIFFUSION SMOOTHING}

;pro-file IDLLevy.pro

;APPLY BY TYPING '. run IDLLevy' in IDL

;ASKS FOR INPUT ZERO PADDED 2NX2N 8bit GREYSCALE TIFF IMAGE

; IDENTIFIES AND PRINTS SIZE OF INPUT IMAGE

;RETURNS USER SELECTED OPTIMAL SMOOTH IMAGE IN FILE 'Levysmooth.tiff'

;RETURNS PROGRESSIVELY SMOOTHER 6 IMAGE SUITE IN FILE 'LevyEvol.tiff'

file1 $='$ '

$\mathrm{sz}=$, ,

plev $=\prime$,

time $={ }^{\prime}$,

read,'enter filename (e.g. idlsharp.tiff): ', file1

image=read_tiff $(f i l e 1)$

ssz=size (image)

$\mathrm{x} 1=\operatorname{ssz}(1)$

$\mathrm{y} 1=\operatorname{ssz}(2)$

print, 'the size of this array is ', $\mathrm{x} 1,{ }^{\prime}{ }^{\prime}, \mathrm{y} 1$

$\mathrm{sz}=\mathrm{x} 1$

read,'enter Levy exponent value p, (e.g. 0.2):', plev

read,'enter final time of smoothing, (e.g. 0.3 ): ', time

close, 1

openu, 1 , file1

$\mathrm{a}=\operatorname{assoc}(1, \operatorname{bytarr}(\mathrm{sz}, \mathrm{sz}, /$ nozero $))$

$\mathrm{B}=\mathrm{a}(0)$

$\mathrm{SB}=\operatorname{Size}(\mathrm{B}, /$ dimensions $) \& \mathrm{~N}=\mathrm{SB}[0]$ \& $\mathrm{M}=\mathrm{SB}[1]$

$\mathrm{u}=($ Findgen $(\mathrm{N})-\mathrm{N} / 2) \# \operatorname{Replicate}(1, \mathrm{M})$

$\mathrm{v}=($ Findgen $(M)-M / 2) \# \# \operatorname{Replicate}(1, N)$

window, $\odot$, xsize $=1800$, ysize $=120 \odot$

DEVICE, DECOMPOSED $=\odot$

LOADCT, $\odot$

$B=\operatorname{Reverse}(B, 2)$

$\mathrm{BB}=\operatorname{CONGRID}(\mathrm{B}, 600,600)$ 


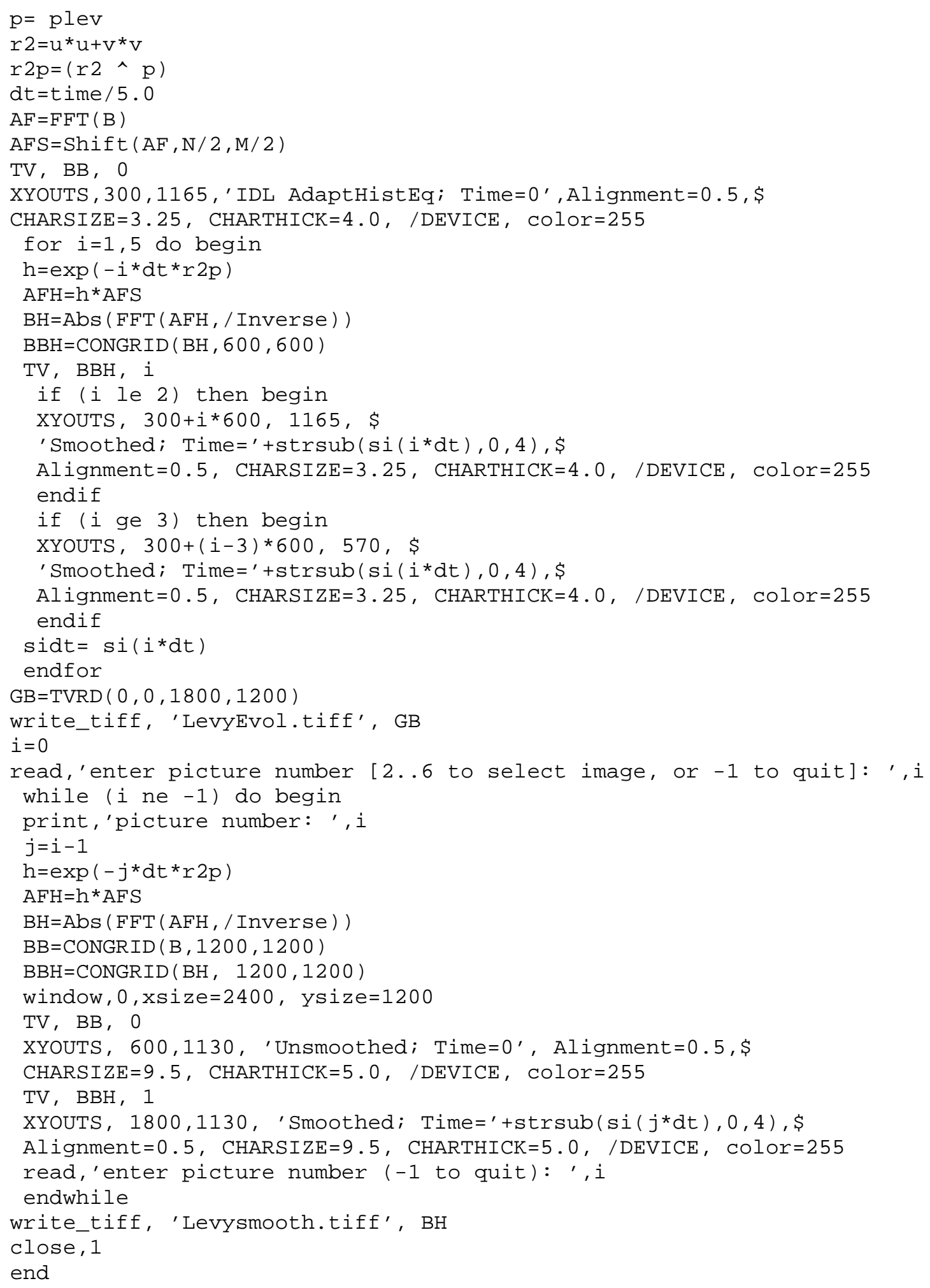




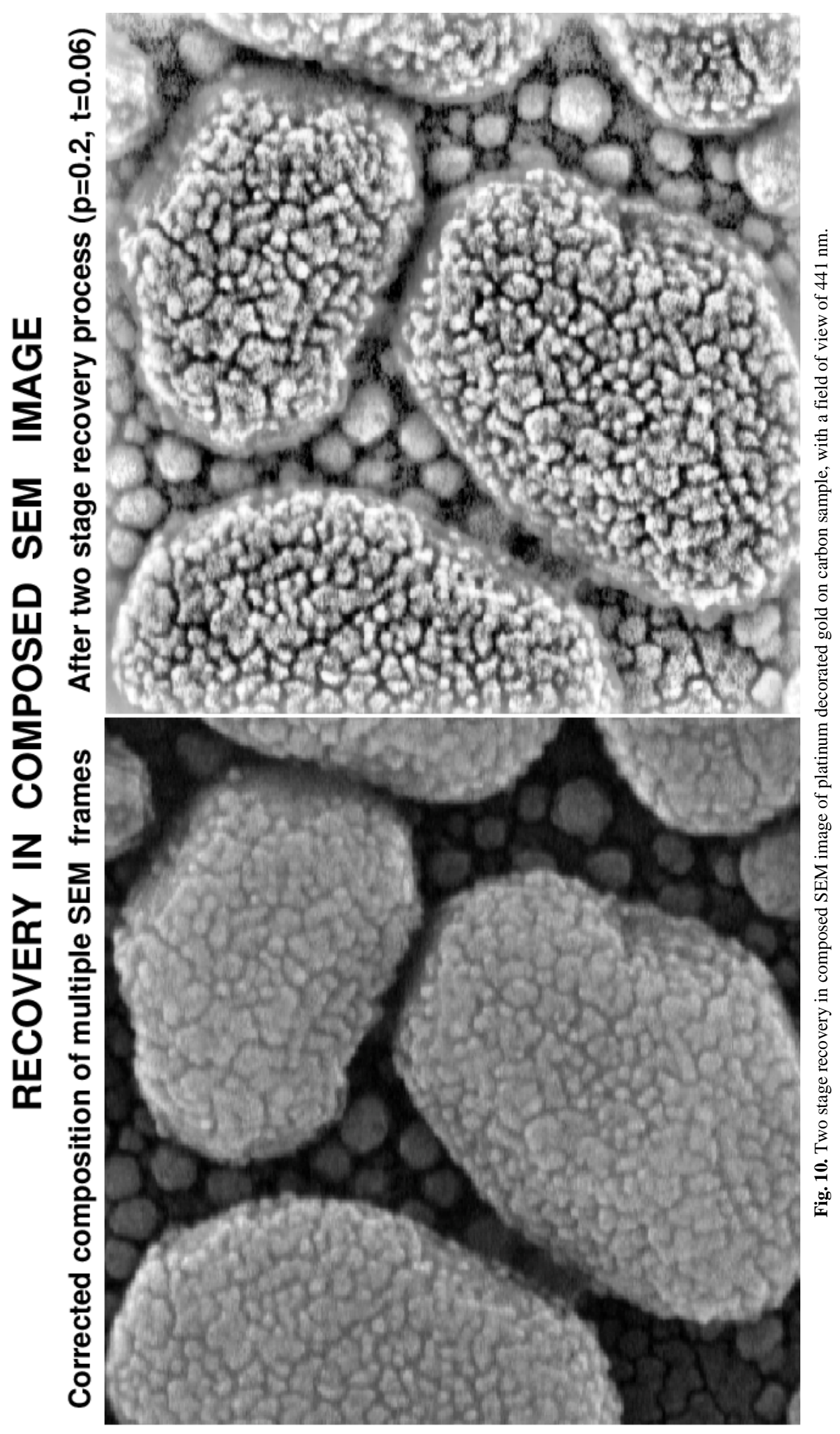




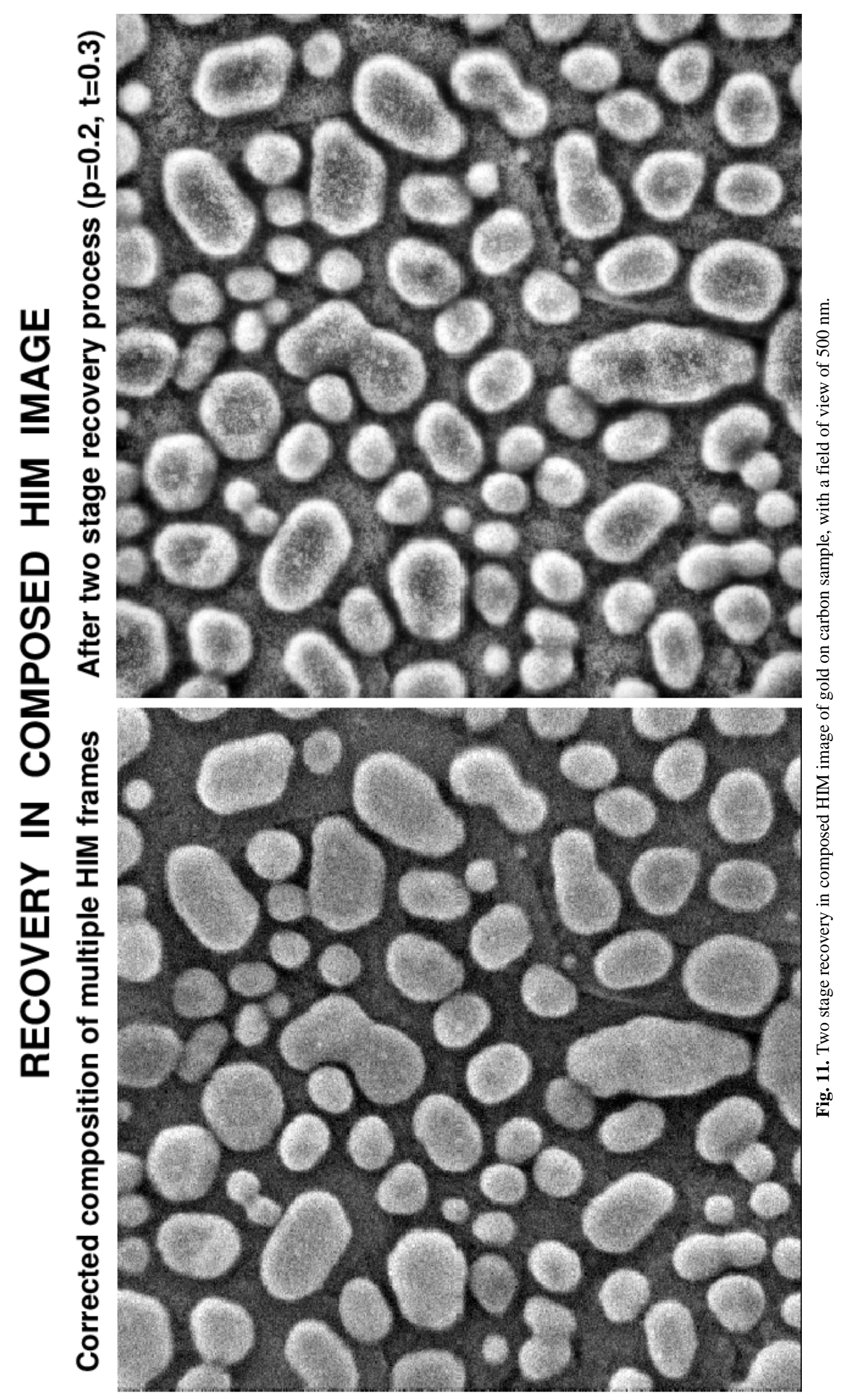




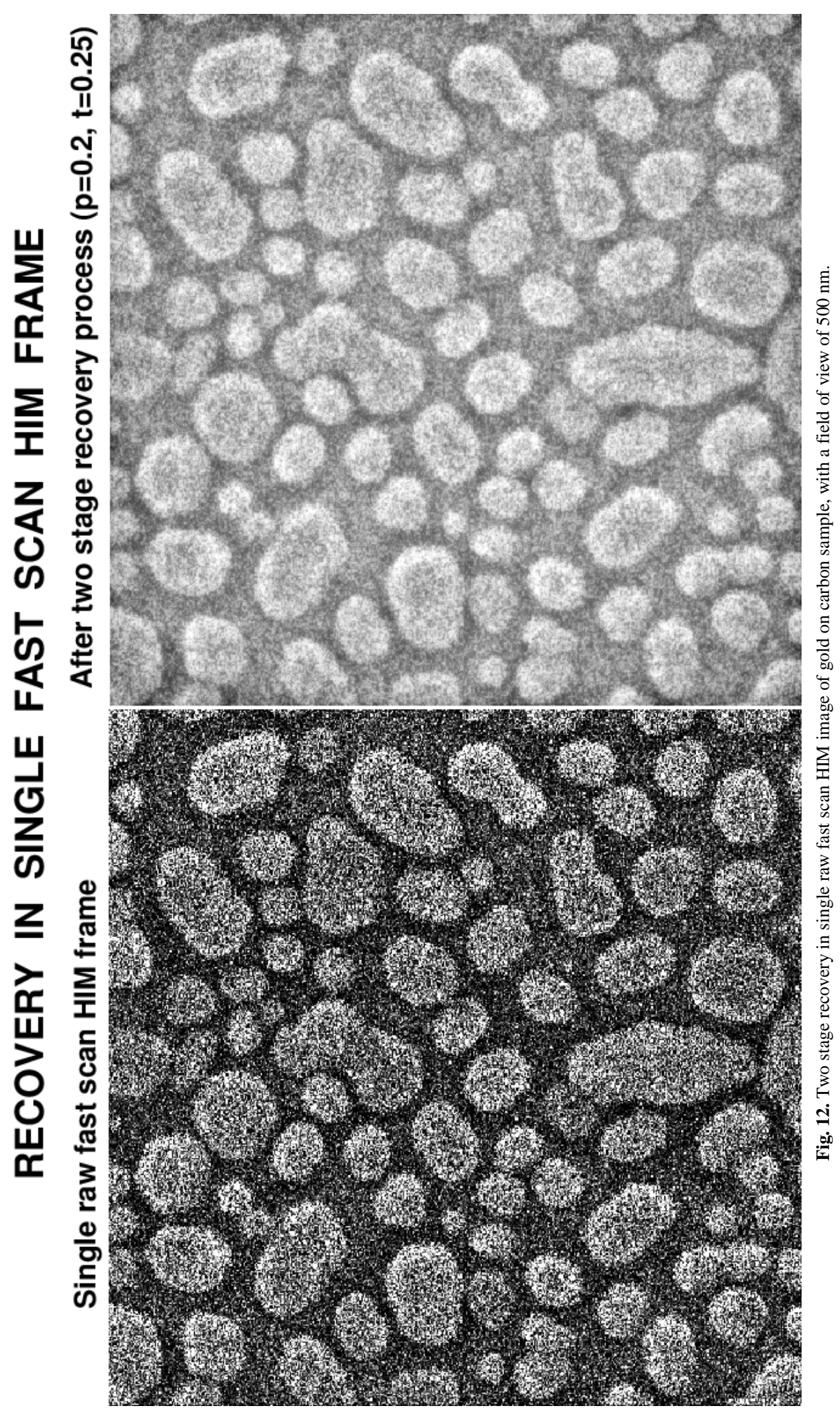




\title{
8. References
}

[1] A. S. Carasso and A. E. Vladár, Fractional diffusion, low exponent Lévy stable laws, and 'slow motion’ denoising of Helium Ion microscope nanoscale imagery, Journal of Research of the National Institute of Standards and Technology, Volume 117 (2012), http://dx.doi.org/10.6028/jres.117.006.

[2] A. S. Carasso, A framework for reproducible latent fingerprint enhancements, Journal of Research of the National Institute of Standards and Technology, Volume 119 (2014), http://dx.doi.org/10.6028/jres.119.006.

[3] P. Cizmar, A. E. Vladár, and M. T. Postek, Real-time scanning charged-particle microscope image composition with correction of drift, Microscopy and Microanalysis, 17 (2010), pp. 302-308, DOI:10.1017/S1431927610094250.

[4] P. Cizmar, A. E. Vladár, and M. T. Postek, Advances in modeling of scanning charged-particle microscopy images, Proc. SPIE 7729 7729OZ (2010), DOI:10.1117/12.861064.

[5] P. Cizmar, A. E. Vladár, and M. T. Postek, Advanced image composition with intra-frame drift correction, Proc. SPIE 8036 80360D (2011), DOI:10.1117/12.887183.

[6] T. Goldstein and S.Osher, The split Bregman method for $L^{1}$ regularized problems, SIAM J. Imaging Sci. 2, 323-343 (2009). See also http://www.math.ucla.edu/ tagoldst/code.html.

[7] ITTVIS Interactive Data Language (IDL), http://www.exelisvis.com/IDL.aspx.

[8] A. Marquina and S. Osher, Explicit algorithms for a new time dependent model based on level set motion for nonlinear deblurring and noise removal, SIAM J. Sci. Comput. 22, 387-405 (2000). http://dx.doi.org/10.1137/S1064827599351751

[9] M. T. Postek and A. E. Vladár, Helium ion microscopy and its application to nanotechnology and nanometrology, Scanning, 30 (2008), pp. 457-462. http://dx.doi.org/10.1002/sca.20129

[10] M. T. Postek, A. Vladár, C. Archie, and B. Ming, Review of current progress in nanometrology with the helium ion microscope, Meas. Sci. Technol 22 (2011), pp. 1-14. http://dx.doi.org/10.1088/0957-0233/22/2/024004

[11] W. K. Pratt, Digital Image Processing, (2012), Wiley-Interscience, New York.

[12] G. Samorodnitsky and M. S. Taqqu, Stable Non-Gaussian Random Processes: Stochastic Models with Infinite Variance, Chapman and Hall, New York, 1994.

[13] R. Sivakumar, Denoising of computer tomography images using curvelet transform, ARPN Journal of Engineering and Applied Sciences 2 (2007), pp. 21-26.

[14] J. L. Starck, E. J. Candès, and D. L. Donoho, The curvelet transform for image denoising, IEEE Transactions on Image Processing, 11, 670-684 (2002). http://dx.doi.org/10.1109/TIP.2002.1014998

\begin{abstract}
About the authors: Alfred S. Carasso received his Ph.D degree in mathematics at the University of Wisconsin in 1968; he was a professor of mathematics at the University of New Mexico, and a visiting staff member at the Los Alamos National Laboratory, prior to joining NIST in 1982. His major research interests lie in the theoretical and computational analysis of ill-posed continuation problems in partial differential equations, together with their application in image reconstruction and computer vision. András E. Vladár is the leader of the Scanning Electron Microscope Metrology (SEM) Project at the National Institute of Standards and Technology (NIST); he received his M.S. and Ph.D. in Electrical Engineering from the Technical University in Budapest, Hungary. Dr. Vladár's research interests are the development of accurate three-dimensional nanometer-scale metrology based on sophisticated SEM instrumentation and measurement methods, and the application of these methods to scientific and technical problems. The National Institute of Standards and Technology is an agency of the U.S Department of Commerce.
\end{abstract}

